\title{
Analog Laboratory Experiments of Pyroclast Surface Impact
}

\author{
JOHN S. ALLEN ${ }^{1}$ RAFSAN RABBI ${ }^{2}$ TADD TRUSCOTT ${ }^{3}$
}

${ }^{1}$ Department of Mechanical Engineering University of Hawaii-Manoa, Honolulu, HI alleniii@hawaii.edu

${ }^{2}$ Dept.of Mechanical Engineering, Utah State University, Logan, UT

${ }^{3}$ Dept.of Mechanical Engineering, Utah State University, Logan, UT

Fluid pyroclasts may undergo a variety of deformations upon impact depending in part on their speed, density and viscosity. These have been observed in field in fire fountains from Hawaiian stye eruptions. Furthermore the extent that the fluid particles under go coalesence or aggultination provides further classification. Previously, laboratory experiments [1] using golden syrup have been developed as analog system. The impact of syrup drops of viscosities $0.04-0.65 \mathrm{~Pa} \mathrm{~s}, 1.0-$ $4.0 \mathrm{~mm}$ diameter, from heights $0.5-1.0$ meter have been investigated upon solid surfaces. Regimes of either deposition or splashing have been identified. In deposition, the drop undergoes a radially spreading in development of a thin lamella and rim on the edge. The drop does not detach from the surface upon receding. Splashing results in secondary droplets which may be ejected during impact or detach from the rim during expansion. In the case of corona splashing, a secondary droplet interacts with thee liquid film of primary droplet. The effects of surface temperature, and impact of liquid pools rather than solid surface were not examined.

We report on different regimes which occur from the impact of drops on liquid pools which are in contact with hydrophobic or hydrophilic surfaces. Modeling pyroclasts which have visco-elastic properties, the impact of gel and nylon spheres (12.5 mm, $17.5 \mathrm{~mm}$ diameter) on liquid pools is also investigated. In the hydrophobic case at low heights $(<35 \mathrm{~cm})$, the spreading and rebound of the visco-elastic gel sphere results in the attachment and lift-off of the liquid in a disc like configuration while heights on the order of $400 \mathrm{~cm}$ the liquid pool spreads into a thin sheets which undergoes disingration. Impact characterictics were quantified with a contact microphone and sound with an air microphone both of which were synchronized to high speed Phantom camera for visualization of the impact and rebound. In a related study, surface temperature is considered for impacting corn syrup drops in terms of spreading and translation.

[1] J. M. Sumner et. al., Journal of Volcanology and Geothermal Research, 142, (2005), 49-65. 